\title{
Preface to the Global Earthquake Forecasting System (GEFS) special issue: Towards using non-seismic precursors for the prediction of large earthquakes
}

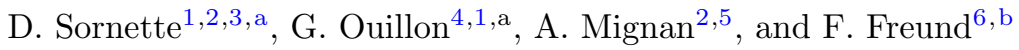 \\ ${ }^{1}$ ETH Zurich, Department of Management, Technology and Economics (D-MTEC), Zurich, \\ Switzerland \\ ${ }^{2}$ Institute of Risk Analysis, Prediction and Management, Academy for Advanced Interdis- \\ ciplinary Studies, Southern University of Science and Technology (SUSTech), Shenzhen, \\ P.R. China \\ ${ }^{3}$ Institute of Innovative Research, Tokyo Institute of Technology, Tokyo, Japan \\ ${ }^{4}$ Lithophyse, Nice, France \\ ${ }^{5}$ Department of Earth and Space Sciences, Southern University of Science and Technology \\ (SUSTech), Shenzhen, P.R. China \\ ${ }^{6}$ GeoCosmo Science Center, NASA Ames Research Park, Moffett Field, USA
}

Received 2 October 2020 / Accepted 7 October 2020

Published online 19 January 2021

Earthquake predictability research has been dominated by the field of Statistical Seismology in the past decades [1], which is exemplified by the relatively recent Collaboratory for the Study of Earthquake Predictability (CSEP, http://www. cseptesting.org) [2], a commendable international effort and well-defined framework. Time-dependent forecasting methods are rather good at predicting seismicity rates (especially aftershocks rates) but fall short in anticipating correctly the magnitudes, especially of large-to-great events [3], and thus fail to predict the bursts of seismic activity immediately associated with large earthquakes. In regard to aftershocks, operational forecasting is possible based on Omori's law ([2] and references therein), but with probabilities only high enough for action in the aftermath of potentially damaging mainshocks [4], hence significantly decreasing their practical value [5]. Another branch of research independently emerged in the 1990s, pushing forward analogies between the many power-laws observed in the phenomenology of earthquakes and the dynamics of critical phase transitions $[6,7]$. This approach led to consider the time of large events as critical points, thus suggesting some predictability especially for large earthquakes, implying that the larger the event, the better their predictability. While this concept seemed to apply well in the laboratory or to small-scale systems such as mines [8] and under stress-controlled conditions, its applicability to large scale, strain-controlled systems such as tectonic plates is still debated $[9,10]$. Other approaches based on pattern recognition are also in use but do not provide really convincing results yet, as they have not been thoroughly tested in

\footnotetext{
a These authors contributed equally.

b e-mail: friedemannf@yahoo.com
} 
real time. The Russian team around Keilis-Borok and Kossobokov has developed a rigorous testing framework (http://www.mitp.ru/en/predictions.html) [11], but the spatiotemporal scale of the prediction range, while of scientific interest, would need significant refinements for any societal impact.

Before and well after Seismology developed into a hard science, countless reports of precursory phenomena have been accumulated ranging from earthquake lights or fluctuations of the electromagnetic fields, as recorded on the ground or by satellites, to abnormal animal behavior. Those precursory signals are thought to reflect timevarying processes associated with the slow tectonic stress accumulation in the Earth's crust. Unfortunately, the Statistical Seismology community never considered such precursors as being reliable or meaningful, which led to a disconnect between the two communities. Their main concerns were: (i) non-seismic precursors were not properly tested in a statistical sense, since the published cases mostly referred to isolated events and were presented without much quantification of the errors (false alarms and missed events); (ii) the reported phenomena displayed a wide diversity but no clear physical model had been formulated that could convincingly explain them in a coherent fashion. The latter argument is strongly reminiscent of Wegener's 1915 continental drift theory that was not accepted by the geophysical community until the 1960's when the fundamental process of mantle's convection had been recognized.

The Global Earthquake Forecast System (GEFS) aims at overcoming this Wegenerian bottleneck by relying on a rather novel theory recently developed in the field of solid-state physics. Based on decades of research, one of us (Freund [12]) has derived a credible, unifying theory for a physical mechanism that is capable of providing explanations for the multitude of reported pre-earthquake phenomena mentioned above. A synthesis has emerged that all pre-earthquake phenomena can be traced back to one fundamental physical process: the activation of electronic charges (electrons and positive holes) in rocks by the activation or break-up of peroxy defects during the ever-increasing tectonic stresses prior to any seismic activity. The positive holes are unusual inasmuch as they are able to flow out of the stressed rock volume, into and through surrounding unstressed or less stressed rock, forming electric currents, traveling fast and far, and causing a wide range of secondary physical and chemical processes along the way. These processes range from electrical ground potentials, stimulated infrared emission, and massive air ionization, to increased levels of carbon monoxide and ozone. The theoretical framework provided allows one to rationalize previous analyses of satellite and ground station data that were recorded before large earthquakes. These recordings provide some evidence that precursory signals tend to become measurable days, sometimes weeks before the disasters. It is worth noting that Freund's theory is not incompatible with seismicity-based theories. They are in fact complementary to each other, proving the untapped diversity of earthquake prediction frameworks.

Since the peroxy defects theory provides a credible hypothesis to understand how the various pre-earthquake signals may be generated, we have a strong rationale for a concerted initiative to continually monitor the Earth's surface, both from ground stations and from satellites, with the goal of covering all relevant possible diagnostics. The appearance of different precursory signs needs to be consolidated in data centers, where data processing, analyses and synthesis will be carried out. A crucial novelty is to use the multi-phenomena, multi-dimensional and multi-scale inputs to obtain robust earthquake alarms. This must be based on rigorous statistical and machine learning techniques designed to tackle sparse intermittent multidimensional data. A strong emphasis on continuous statistical testing of the relevance and confidence of the precursors need to be developed to assess and continue to improve the performance of the forecasts. Such an initiative has only become feasible in recent years thanks to new technological development in computing power and in big data 
management. The GEFS is a collaborative initiative that aims to transform the field of earthquake science by building a coherent edifice of signals for reliable earthquake forecasts. It has the potential to become a cornerstone for the development of timedependent preparatory measures to protect sensitive infrastructures and the population. As is easily apparent, the success of efforts like the proposed GEFS will largely rely on the active collaboration of multiple teams within an interdisciplinary framework, involving data acquisition and processing, theoretical developments, numerical and experimental modeling, and a large amount of statistical testing in order to validate the existing theory and emerging hypotheses.

By presenting a collection of works establishing the state-of-the-art in this complex and challenging field, this special issue hopes to be a catalyst for such a wideranging collaboration. The introductory article by Freund et al. [13] provides a review of observed potential precursors in the light of the peroxy defects theory. It is followed by twenty studies, which are further analyzed and discussed in the concluding article by Mignan et al. [14]. Works that are part of this special issue, listed by alphabetical order, are the following: Anagnostopoulos et al. [15] investigate the correlation between solar activity and large-to-great earthquake occurrences over several solar cycles. Bhardwaj et al. [16] present their view on the challenges and possibilities of earthquake prediction using non-seismic precursors. Chen et al. [17] investigate geoelectric signals as potential precursors to large earthquakes for which Chen et al. [18] provide a physical framework based on Freund's peroxy defects theory. Mansouri Daneshvar and Freund [19] explore the possible relationship between precipitation and earthquakes. Freund et al. [20] investigate unipolar electromagnetic pulses in the context of the peroxy defects theory. Kalenda and Neumann [21] use a network of subsurface inclinometers to investigate whether horizontal deformation prior to great earthquakes could also be detected. Kamer et al. [22] showcase Richter-X, a publicly accessible web-based platform that allows one to test prospectively any seismic forecasting statement. Lai et al. [23] examine changes in both groundwater temperature and water level as potential precursors to large earthquakes. Meng and Zhang [24] explore the potential relationship between thermal and methane anomalies associated to the 12, May 2008 M8.0 Wenchuan earthquake, China. Nandan et al. [25] present state-of-the-art Epidemic Type Aftershock Sequence (ETAS) models used for null-hypothesis testing in Richter-X. Nemec et al. [26] investigate the possible link between hundreds of earthquakes and very low frequency (VLF) signals observed by the DEMETER satellite. Parrot et al. [27] explore various atmospheric and ionospheric anomalies prior to large earthquakes. Piroddi [28] describes how precursory signals of seismic activity might be found in geostationary thermal infrared (TIR) data. Potirakis et al. [29] review the literature of potential electromagnetic precursors to large earthquakes (fracto-electromagnetic emissions, ultra-low frequency (ULF), and sub-ionospheric VLF). Qin et al. [30] investigate the association of two signals, groundwater temperature and radon, prior to two large-to-great earthquakes in China. Scoville and Freund [31] test the possible origin of TIR anomalies in laboratory experiments. Yan et al. [32] compare ULF electric data recorded on ground stations and at the low-altitude DEMETER satellite as a sanity check for future earthquake precursor analyses. Zhang et al. [33] reproduce a past study on TIR anomalies and obtain new results based on improved statistical tests. Zhuang et al. [34] discuss the critical point theory in which large earthquakes may be forecasted in probabilistic terms and compare different forecasting methods. This list proves the diversity of precursors covered in this volume. Various pattern recognition methods are presented and illustrated with examples taken from specific sites to global datasets. The reported observations cover various regions of the world.

The proposed GEFS is articulated around a theoretical core, proposed by one of us (Freund [12]) and co-workers [13]. This volume thus offers an excellent opportunity 
to discuss reported observations that can be accounted for within this framework, discuss agreements and possible discrepancies. The reported observations stand also on their own merit independently of theory and provide the motivations for further observations, testing, and understanding towards operational use in forecasting. Importantly, the GEFS represents a first step to build an efficient community to share resources such as inventories of existing databases relative to ground-based or remote observations, to share assessments which databases provide quantitative information and which ones are more qualitative, and finally identify methodologies to reveal anomalies (here defined as low-probability fluctuations above a background signal). Some of them just rely on the relative raw amplitudes of the observed signal, while others use preliminary second-order statistical pre-processing (such as the computation of associated statistical moments). It is our hope that this special issue will reconcile the different scientific communities with the shared goal to better forecast, and ultimately predict, large earthquakes.

Publisher's Note The EPJ Publishers remain neutral with regard to jurisdictional claims in published maps and institutional affiliations.

\section{References}

1. D. Vere-Jones, Y. Ben-Zion, R. Zuniga, Pure Appl. Geophys. 162, 1023 (2005)

2. D. Schorlemmer, et al., Seismol. Res. Lett. 89, 1305 (2018)

3. A. Helmstetter, D. Sornette, J. Geophys. Res. 108, 2482 (2003)

4. P.A. Reasenberg, L.M. Jones, Science 265, 1251 (1994)

5. A. Mignan, Sci. Rep. 4, 4099 (2014)

6. D.D. Bowman, G. Ouillon, C.G. Sammis, A. Sornette, D. Sornette, J. Geophys. Res. 103, 24359 (1998)

7. K.F. Tiampo, M. Anghel, Tectonophys. 413, 1 (2006)

8. G. Ouillon, D. Sornette, Geophys. J. Int. 143, 454 (2000)

9. A. Mignan, Tectonophys. 505, 1 (2011)

10. A. Mignan, J. Seismol. 23, 771 (2019)

11. V. Keilis-Borok, Annu. Rev. Earth Planet. Sci. 30, 1 (2002)

12. F.T. Freund, Acta Geophys. 58, 719 (2010)

13. F. Freund, G. Ouillon, J. Scoville, D. Sornette, Eur. Phys. J. Special Topics 230, 7 (2021)

14. A. Mignan, G. Ouillon, D. Sornette, F. Freund, Eur. Phys. J. Special Topics 230, 473 (2021)

15. G. Anagnostopoulos, I. Spyroglou, A. Rigas, P. Preka-Papadema, H. Mavromichalaki, I. Kiosses, Eur. Phys. J. Special Topics 230, 287 (2021)

16. A. Bhardwaj, L. Sam, F.J. Martin-Torres, Eur. Phys. J. Special Topics 230, 367 (2021)

17. H.-J. Chen, C.-C. Chen, G. Ouillon, D. Sornette, Eur. Phys. J. Special Topics 230, 381 (2021)

18. H.-J. Chen, C.-C. Chen, G. Ouillon, D. Sornette, Eur. Phys. J. Special Topics 230, 67 (2021)

19. M.R. Mansouri Daneshvar, F.T. Freund, Eur. Phys. J. Special Topics 230, 335 (2021)

20. F.T. Freund, J.A. Heraud, V.A. Centa, J. Scoville, Eur. Phys. J. Special Topics 230, $47(2021)$

21. P. Kalenda, L. Neumann, Eur. Phys. J. Special Topics 230, 353 (2021)

22. Y. Kamer, S. Nandan, G. Ouillon, S. Hiemer, D. Sornette, Eur. Phys. J. Special Topics 230, 451 (2021)

23. G. Lai, C. Jiang, W. Wang, L. Han, S. Deng, Eur. Phys. J. Special Topics 230, 275 (2021)

24. Q. Meng, Y. Zhang, Eur. Phys. J. Special Topics 230, 247 (2021)

25. S. Nandan, Y. Kamer, G. Ouillon, S. Hiemer, D. Sornette, Eur. Phys. J. Special Topics 230, 425 (2021) 
26. F. Nemec, M. Liska, M. Parrot, Eur. Phys. J. Special Topics 230, 227 (2021)

27. M. Parrot, V. Tramutoli, T.J.Y. Liu, S. Pulinets, D. Ouzounov, N. Genzano, M. Lisi, K. Hattori, A. Namgaladze, Eur. Phys. J. Special Topics 230, 197 (2021)

28. L. Piroddi, Eur. Phys. J. Special Topics 230, 111 (2021)

29. S.M. Potirakis, Y. Contoyiannis, A. Schekotov, K. Eftaxias, M. Hayakawa, Eur. Phys. J. Special Topics 230, 151 (2021)

30. K. Qin, Z. Shuo, L. Wu, Y. Wang, Eur. Phys. J. Special Topics 230, 263 (2021)

31. J. Scoville, F. Freund, Eur. Phys. J. Special Topics 230, 85 (2021)

32. R. Yan, L. Wang, M. Parrot, X. Zhang, Z. Hu, Eur. Phys. J. Special Topics 230, 179 (2021)

33. Y. Zhang, Q. Meng, G. Ouillon, L. Zhang, D. Hu, W. Ma, D. Sornette, Eur. Phys. J. Special Topics 230, 133 (2021)

34. J. Zhuang, M. Matsu'ura, P. Han, Eur. Phys. J. Special Topics 230, 409 (2021) 